\title{
المادة التعليمية للغة العربية على أسس قيم الألعاب التقليدية بإندونيسيا
}

\section{Nur Lailia Rahmawati}

Sekolah Tinggi Agama Islam At-Tanwir Bojonegoro

lailaabdullah899@gmail.com

DOI: http://dx.doi.org/10.18326/lisania.v2i1.44-61

\begin{abstract}
This research aims to develop Al-'Arabiyyah Li al-Athfâl (ALA) Learning Materials based on Indonesian Traditional Games for students of Arabic Education Department STAI At-Tanwir Bojonegoro by producing book and to know the validity and feasibility of Al-'Arabiyyah Li -Athfâl (ALA) based on Indonesian Traditional Games for students of students of Arabic Education Department STAI At-Tanwir Bojonegoro Arabic Study Program. The type of research used is research and development of ADDIE (Analyze, Design, Develop, Implement, Evaluate) model. The types of data include quantitative and qualitative, while data collection instruments used are questionnaires and interviews and to be analyzed by using descriptive and qualitative statistical analysis. The results show that book produced is valid and feasible to be used in Arabic learning process, seen from expert test analysis and user response test.
\end{abstract}

Keywords: Arabic Language Materials, Traditional Games of Indonesia, students of Arabic Education Department

\begin{abstract}
Abstrak
penelitian ini bertujuan untuk mengembangkan Bahan Ajar Al-'Arabiyyah Li al-Athfâl (ALA) berbasis Permainan Tradisional Indonesia untuk mahasiswa Prodi Pendidikan Bahasa Arab STAI At-Tanwir Bojonegoro dalam bentuk produk buku dan mengetahui validitas dan kelayakan Bahan Ajar Al'Arabiyyah Li al-Athfâl (ALA) berbasis Permainan Tradisional Indonesia untuk mahasiswa Prodi Pendidikan Bahasa Arab STAI At-Tanwir Bojonegoro. Jenis penelitian yang digunakan adalah penelitian pengembangan (Research \& Development) model ADDIE (Analyze, Design, Develop, Implement, Evaluate). Jenis data meliputi data kuantitatif dan data kualitatif, sedangkan instrumen pengumpul data menggunakan angket dan wawancara serta dianalisis menggunakan analisis statistik deskriptif dan kualitatif. Hasil penelitian menunjukkan bahwa produk buku ini valid dan layak digunakan dalam proses pembelajaran bahasa Arab, dilihat dari analisis uji ahli serta uji tanggapan pengguna.
\end{abstract}

Kata Kunci: Bahan Ajar Bahasa Arab, Permainan Tradisional Indonesia, Program Studi Pendidikan Bahasa Arab 
المواد التعليمية تعد من أهم العناصر فن نجاح عملية تعليمية. المواد التعليمية هي كل مـا يريـد المعلـم إيصـالها مـن المعلومـات إلى أذهـان الطلاب عنــ عمليـة التعليم أي المحتـوى التعليمي الـذي يرغـب في تقديمـه بغـرض تحقيـق أهـاف تعليميـة معرفيـة، وهـي المضمون الذي يتعلمه الطلاب في علم ما. ويقول رشدى أحمد طعيمة بأن المواد التعليمية

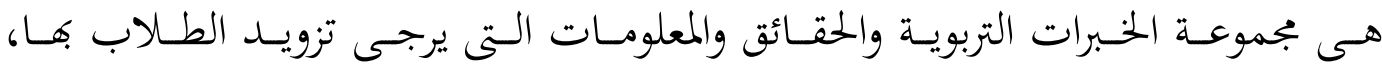
والابحاهات والقيم التى يراد تنميتها عندهم، أو المهارات الحركية التى يراد اكسابها إياهم، يهدف تحقيق النمو الشـامل المتكامل لهم فن ضوء الأهـاف المقررة في المنهج (طعيمة، $\cdot(r \cdot r: r \cdot r$

إن المـواد التعليميـة لمـا أهميـة كبـيرة لـدى المدرسـين في تعليم اللغـة العربيـة، لأكها تساعدهم في تعليمهم، وكذلك لها أثر كبير لدى الطلبة في تعلم اللغة العربية وتساعدهم في مجتمعـاةم الـتي ينتمـون إليهـا لتكـوين الاسـتعدادات والقـدرات للتعبـير عـن أفعـالهم المحدودة وتعلم المعلومات وتقديمها باللغة العربية دون تفكير في اللغة نفسها. ولقـد استخدم مصطلح الألعاب في تعليم اللغـة، لكي يعطى ججالا واسعا مـن الأنشـطة الفصـلية التي تسـتخدم في التـــريب على اسـتخدام اللغـة وممارسـتها، وهـــهـ الألعاب تخضع لإشراف المعلم وتوجيهه، حيث تسهم هذه الألعاب في تيسير عملية تعلم اللغة في شكل لعبة محكومة بقواعد معينة. والألعاب اللغوية هي "نشاط يتم بين الدارسين للوصول إلى غايتهم في إطار قواعد موضوعة" أو بعبارة أخرى هي" مجموعة من الأنشطة الفصلية التي تهدف إلى تزويد المعلم والمتعلم بوسيلة متعة ومشوقة للتدريب على عناصر اللغة، وتوفير الحوافز لتنمية المهارات اللغوية المختلفة في إطار قواعد موضوعية تخضع لإشراف المعلم أو لمراقبته على الأقل . وتعـرف الألعـاب اللغويـة إجرائيـا بأفها "نشـاط موجـه يقـوم بـه التلاميـ فرديا أو جماعيا وفق قواعد متفق عليها وتمتاز بالسرعة والحركة والتنافس، وتهدف إلى الاستمتاع وفهم المعلومات". ومن ناحية أخرى فهي "نشاط يتم بين الدارسين بشكل إرادي، يؤدى في حسدود زمـان ومككان معينـين حسب قواعـد مقبولة، وبتوجيـه مـن المعلم (مصـفىى, 


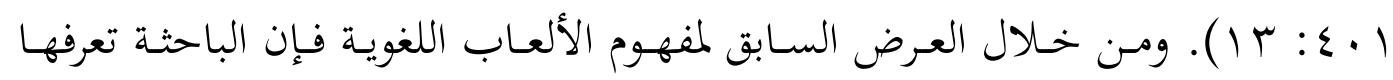

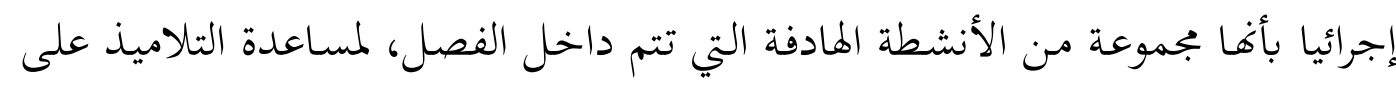
التعلم اللغوي الجيد، ولتحقيق أهداف لغوية محددة.

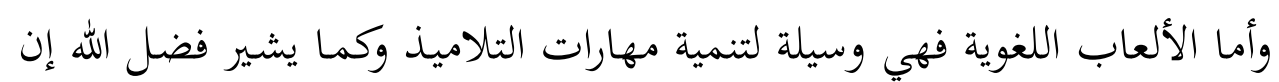

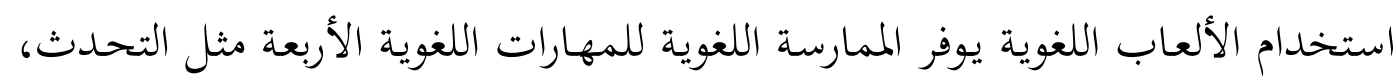

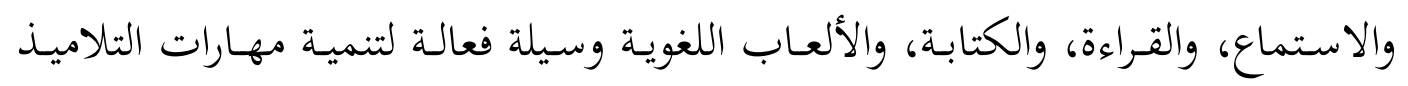

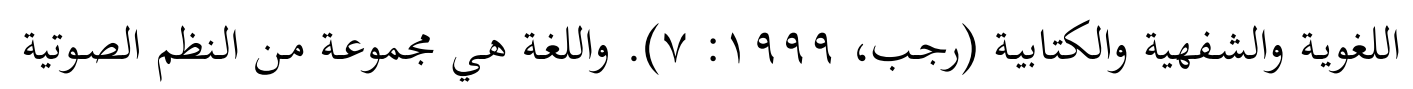

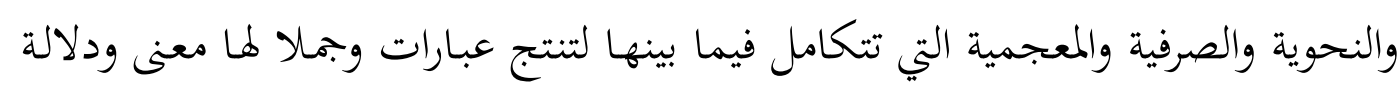



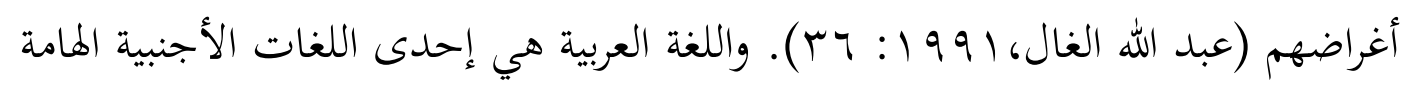
في العالم لاسيما في إندونيسيا، وهي لغـة الدين ولغة الثقافـة الإسلامية ولغة الاتصـال

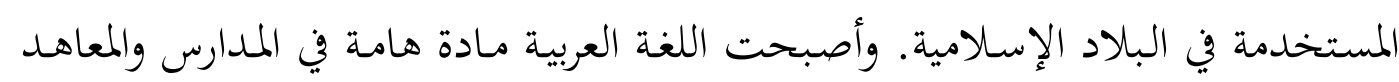

$$
\text { والجامعات الإسلامية بجانب الدروس الأخرى. }
$$

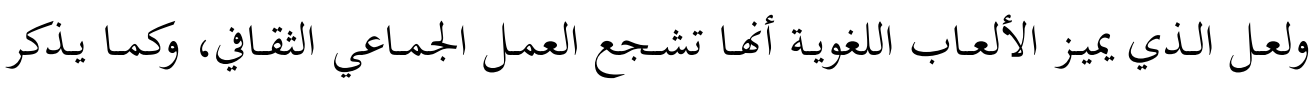

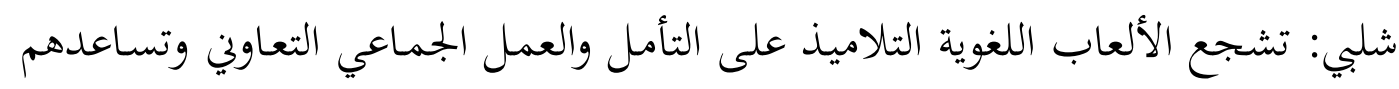

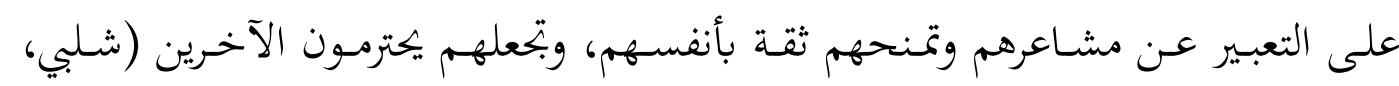

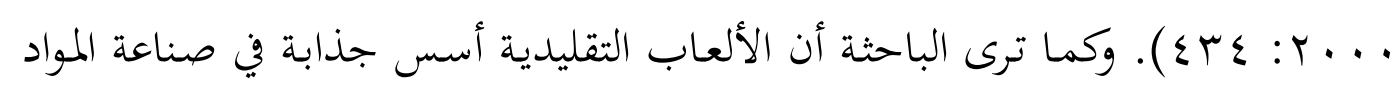

التعليمية.

اعتمادا على ما سبق، كتبت الباحثة هذا البحث تحت العنوان المادة التعليمية على أسس قيم الألعاب التقليدية بإندونيسيا (بحث تطويري لدى طلبة قسم تعليم اللغة

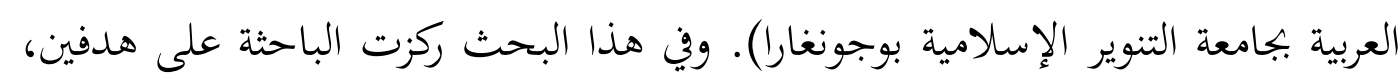

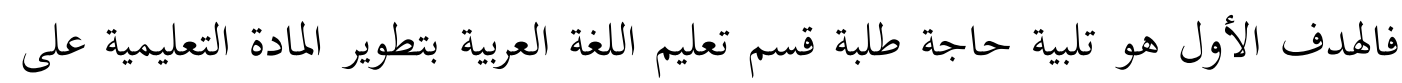

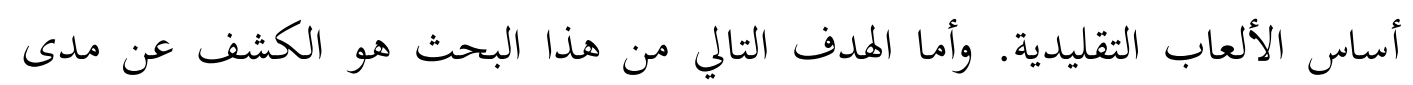
صادق المادة التعليمية وصالحها على أساس الألعاب التقليدية لدى طلبة قسم تعليم اللغة التها

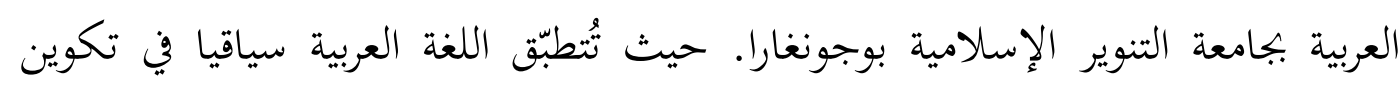


قدرة المتخرجين بالكفاءات التي تحتوي على السلوك والمعلومة والمهارة في مهنة تعليم اللغة العربية .

\section{منهجية البحث}

استخدم هذا البحث منهج البحث والتطوير، وهو طريقة البحث المستخدمة

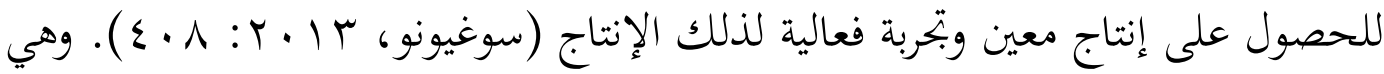
طريقة البحث لإنتاج شيء جديد أو لإتمام شيء معين. وبهذا البحث التطويري


يستخدمها الباحثة في إجراء هذا البحث بطريقة ADDIE. وينقسم هذا البحث إلى خمس بـ خطوات، وهي: الدراسة المبدئية، والتصميم، والتطوير، والتجربة، والتعديل. ومصادر البيانات المستخدمة في هذا البحث هي الكتب التي تضم النظريات المتعلقة

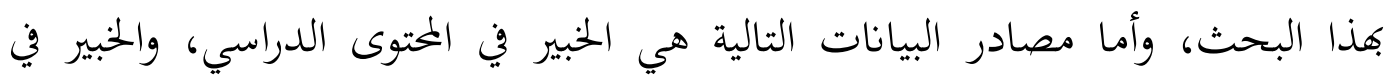
تصميم المادة التعليمية، ومعلمة مادة اللغة العربية في قسم تعليم اللغة العربية والطلبة في

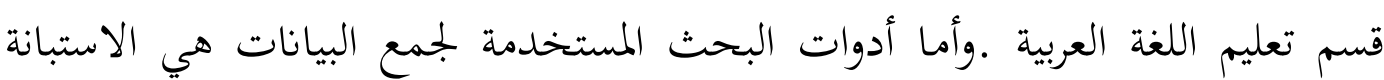
والمقابلة. وتحليل هذه البيانات عن طريق التحليل الوصفي.

\section{مفهوم الألعاب التقليدية في تعليم اللغة العربية}

الألعاب التقليدية فهي اللأنشطة الترويحية التي لعبها المجتمع ويمكنها أن تصبح

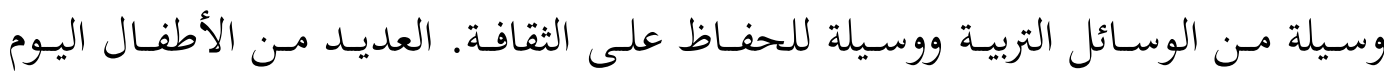

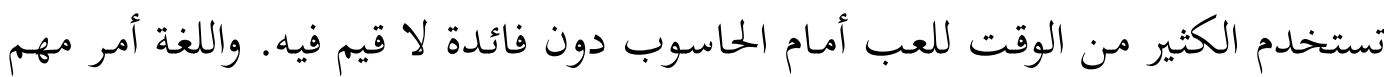
في الألعاب التقليدية لأها وسيلة للإتصال. ونستطيع استخدام الألعاب التقليدية كوسيلة



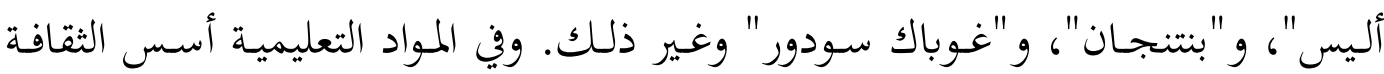

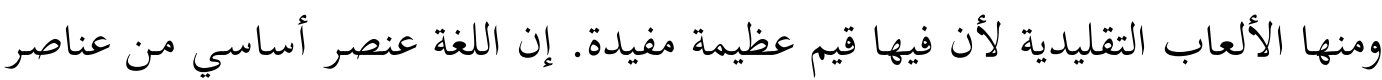

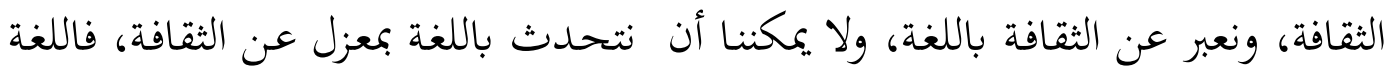


وعاء الثقافة. فتعليم اللغة الأجنبية هو تعليم حضارة أصحاب اللغة أيضا. للذلك لابد لدارس اللغة الأجنبية أن يتعرف على حضارة المجتمع. كما قال وديو نوغراهـا في بكثنه أن القيم في الألعاب التقليدية تنقسم إلى أربعة أقسـام وهي قيم تتعلق بنفسـه و قيم تتعلق بالآخرين و قيم تتعلق بالبيئة و قيم تتعلق بالوطنية. وفيما يلي البيان لكل قسم من الأقسام الأربعة: الجدول ا ـ القيم في الألعاب التقليدية

\begin{tabular}{|c|c|c|}
\hline 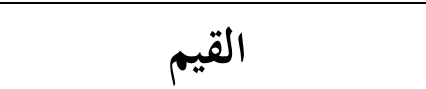 & 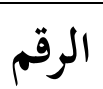 & علاقة القيم \\
\hline الصدق & 1 & \multirow{7}{*}{ القيم التي تتعلق بنفس } \\
\hline المسؤولية & $r$ & \\
\hline صحة الحياة & 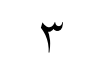 & \\
\hline الانضباط & $\varepsilon$ & \\
\hline 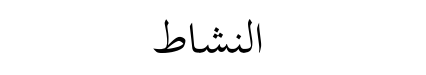 & 0 & \\
\hline الاعتماد على النفس & 7 & \\
\hline مستقل النفس & V & \\
\hline الوعي بالحقوق والواجبات & $\Lambda$ & \multirow{4}{*}{ القيم التي تتعلق } \\
\hline الطاعة بالدستور & 9 & \\
\hline الأدب والتهذيب & $1 \cdot$ & \\
\hline الديمقراطية & 11 & \\
\hline الاهتمام بالبيئة والمجتمع & lr & القيم التي تتعلق بالبيئة \\
\hline الوطنية & Ir & \multirow{2}{*}{ بالقيم التي تتعلق } \\
\hline احترام التنوع & $1 \varepsilon$ & \\
\hline
\end{tabular}




\section{المادة التعليمية للغة العربية على أسس قيم الألعاب التقليدية بإندونيسيا}

إنّ الخطوات في تطوير المادة التعليمية على أساس الألعاب التقليدية فهي كما يلي:

\section{الدراسة المبدئية}

الخطوة الأولى في منهج البحث والتطوير هي الدراسة المبدئية أو تحليل الاحتياجات لنيل المعلومات الأولى. وللحصول عليها فُنّْذت المقابلة مع مدرسة اللغة العربية لمادة اللغة العربية الأولى بقسم تعليم اللغة العربية وهي الأستاذة دوي خيرة النساء الماجستير وطلبة قسم تعليم اللغة العربية. ونتائج هذه المقابلة هي: (1) من جهة عملية التعليم: أنّ أكثر عملية التعليم في هذه المادة هي أنشطة القراءة والكتابة. (Y) من جهة المادة المستخدمة: أنّ المواد مأخوذة من المصادر المتنوعة دون استخدام الكتاب المقرر الخاص. فالموضوعات غير مناسبة بحاجاتم في مجال تعليم اللغة العربية. (ץ) من جهة كفاءة المعلّمة: أنّ الكفاءة أكثرها لترقية مهارة القراءة والكتابة أي هناك قلة التوازن في الحصول على الكفاءات أو المهارات الأربعة وهي المهاءه الاستماع والكلام والقراءة والكتابة. (ع) من جهة إتاحة الفرصة للطلبة: أنّ المعلمة لم تتيح الفرصة لجميع الطلبة لتطوير مهاراتم. (0) من جهة ارتفاع قدرة الطلبة:لم

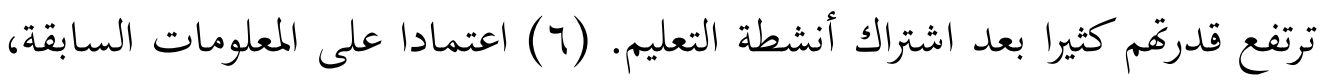
قبل القيام بتصميم المادة التعليمية فالخطوة هي ملاحظة الكتب المتنوعة والمراجع الأخرى المناسبة ومراجعتها ثم تعيين الموضوعات في المادة التعليمية نسبة إلى حاجات

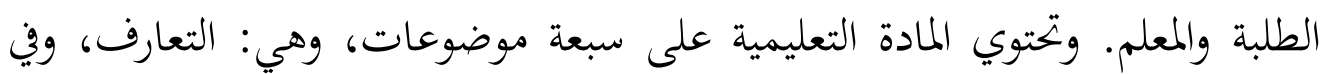
السوق، والبحث عن العمل، ووكالة السفر، وفي البنك، وفي بيت المال، والفن

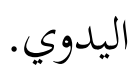

\section{التصميم}

بعد القيام بالدراسة المبدئية ونيل المعلومات الكافية وتعيين الموضوعات فالخطوة الثانية هي التصميم. وفي هذه الخطوة حُدّدت المشكلات المناسبة مع ميدان

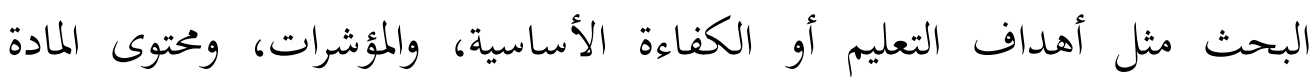
التعليمية، ونموذج عرض المواد، والتدريبات أو التقويم. وعملية تعيين الكفاءة 
الأساسية والمؤشرات أعدّها الباحثة بنفسه لأنّ المنهج الخاص لمادة اللغة العربية بقسم تعليم اللغة العربية غير موجود.

والكفاءة الأساسية هي أنْ تكون للطلبة قدرة على تعبير الآراء والأفكار والشعور شفويا ووتريريا في اللغة العربية عن موضوعات تعليم اللغة العربية السياقية.

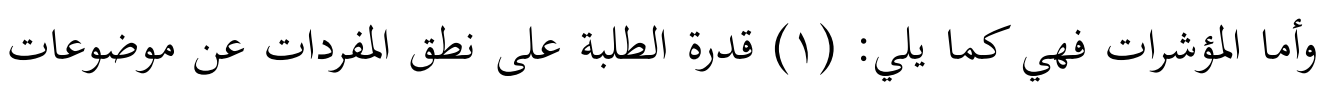

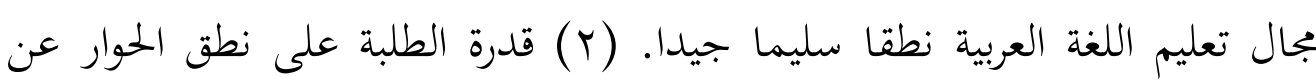
موضوعات بجال تعليم اللغة العربية نطقا صحيحا مناسبا بتنغيم اللغة العربية. (r) قدرة الطلبة على تطبيق الحوار باللغة العربية عن موضوعات مجال تعليم اللغة العربية

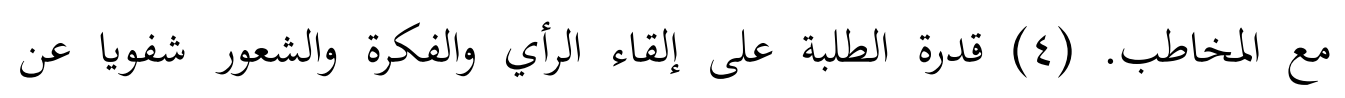

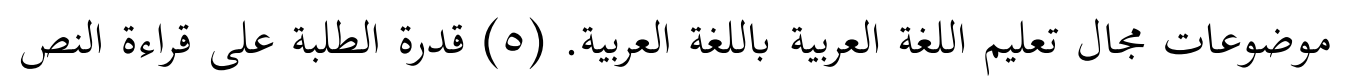

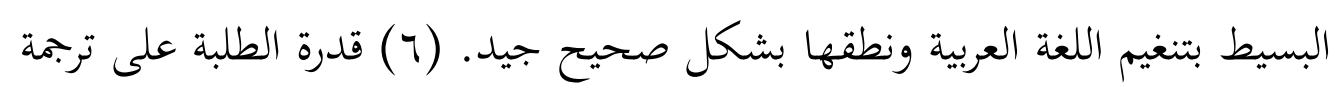
الجملة البسيطة ترجمة صحيحة سليمة. (V) قدرة الطلبة على تلخيص النص المقروء بلغتهم تلخيصا صحيحا حسنا. (م) قدرة الطلبة على إعطاء حركات الكلمات وترتيب الجملة البسيطة بشكل صحيح. (9) قدرة الطلبة على تعبير الرأي والفكرة والشعور عن موضوعات تعليم اللغة العربية كتابة بسيطة صحيحة.

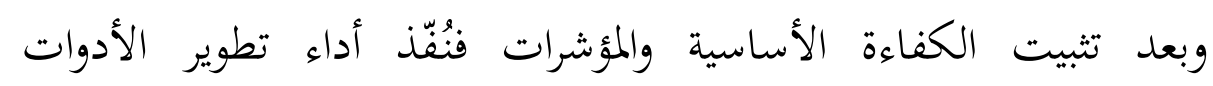
التقييمية. وتتعلق هذه الأدوات بالكفاءة الأساسية المرجوة التي تعتمد على المؤشرات

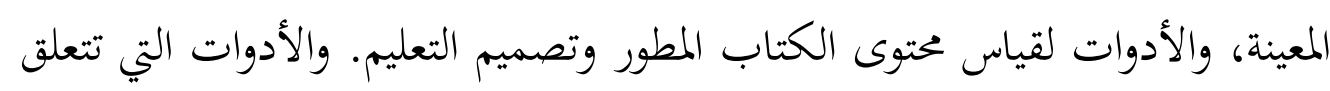

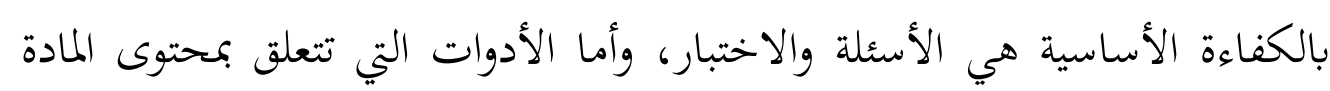
وتصميم التعليم هي الاستبانة.

التطوير

في هذه الحخطوة طُوّرت المادة التعليمية. وأما مواصفات المادة التعليمية المطورة على أساس الألعاب التقليدية فهي كما يلي:

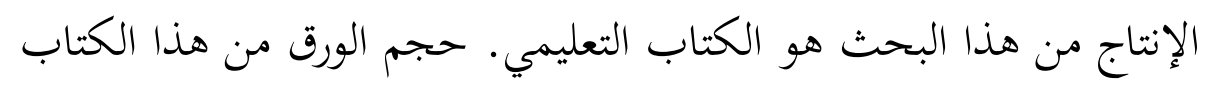

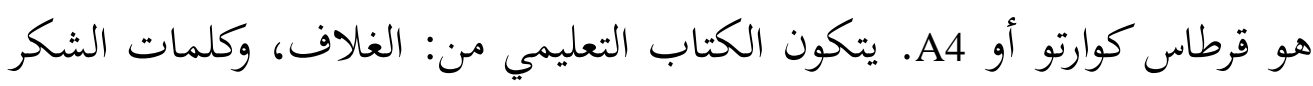


والتقدير، ووصف استخدام الكتاب، والكفاءة الأساسية، والمؤشرات، والفهرس ومحتويات الكتاب.

والأنشطة المستخدمة في تعليم الاستماع منها: يكرر المعلم والطلبة كلمة فكلمة متبادلا فرديا أو جماعيا، يجلس الطلبة في المجموعات ثم يشير كل واحد إلى الصورة ويجيب الآخر عن اسمها متبادلا. والأنشطة المستخدمة في تعليم الحوار منها: يفهم الطلبة الحوار فهما جيدا، يجلس كل الطلبة في المجموعات ثم يطبقون الحوار متبادلا، يبدل الطلبة الحوار لتطور مهارة الكالام، يستخدم الطلبة الحوار مع زملاءهم، يعبر الطلبة القصة من الصور المؤيدة متبادلا ثم يعلقون بعضهم بعضا. والأنشطة المستخدمة في تعليم القراءة منها: يسمع الطلبة النص ويكرر قرائته في المجموعات جهرا وحسنا من جهة التنغيم والنطق، يفهم الطلبة النص بتكوين السؤل والجواب عن النص أو بتكوين السؤال عن الجواب المؤيد ثم يناقشونه، يترجم الطلبة الجمل البسيطة ويناقشوها في المجموعات، يلخص الطلبة النص بلغتهم ثم يناقشون الخلاصة ويتعالقوها في المجموعات. والأنشطة المستخدمة في تعليم الكتابة منها: يفهم الطلبة أسلوب الجملة الصحيحة، يعبر الطلبة أسلوب الجملة بتكوين الجملة الجديدة من الكلمات المؤيدة، يعبر الطلبة الرأي والفكرة والشعور كتابة ثم يقومون بتعليق نتائج الكتابة بعضهم ببعض متبادلا، يعطي الطلبة حركات الكلمات والجمل بشكل صحيح، يرتب الطلبة الجمل المبعثرة ويغيروها إلى جمل كاملة. وقائمة المفردات باللغة العربية مع الصور. يتميز هذا الكتاب بشتى أنواع من حيث التصميم الملوّن والصور المأخوذة من الإنترنت والوثائق الذاتية بتفضيل مناسبة الصور بالموضوع وثقافة الجامعة والمنطقة. يتميز هذا الكتاب بالألعاب التقليدية ، والذي يتمثل في أنّ الكتاب: يعطي الطلبة الفرصة الواسعة للمساهمة في عملية التعليم، يجعل المعلم وسيطا ومشجعا ومخخطا في أنشطة التعليم، يجعل الطلبة عاملين في التعليم السياقي، يجعل التعليم عملية مفيدة ونافعة، يكون أنشطة التعليم عديدة ومتنوعة، يريح الطلبة في 
عملية التعليم، يجعل الطلبة متفاعلين بينهم ببعض وبالمعلم لبناء التفاعل الاجتماعي ومهارة الاستماع والكلام والقراءة والكتابة.

وبعد فاية التصميم وتطوير المادة التعليمية على أساس الألعاب التقليدية

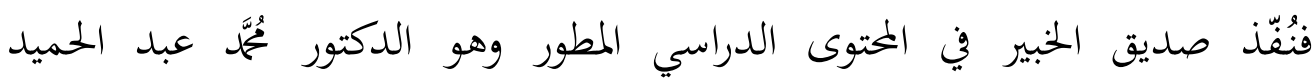
الماجستير معلم في الدراسات العليا بجامعة مولانا مالك إبراهيم الإسلامية الحكومية

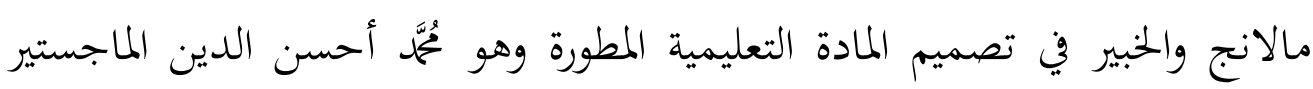
معلم في قسم الأدب العربي بجامعة مالانج الحكومية. بالنسبة إلى مقياس ليكرت (Likert) تتصورت النتيجة من تصديق الخبير في المختوى الدراسي المطور كما يلي:

صورة 1: مقياس ليكرت من نتيجة تصديق الخبير في المحتوى الدراسي المطور

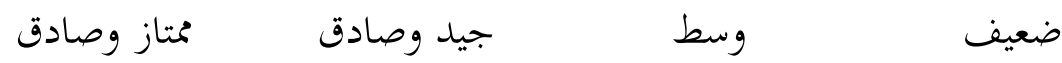

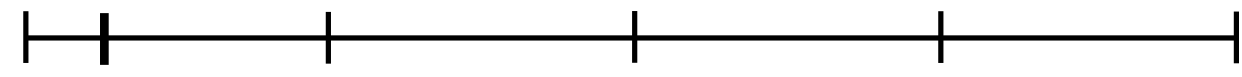

IEA ITr $111 \quad$ VE TV

وفي أداة تصديق الخبير في المحتوى الدراسي Vr أسلوبا بعرض الدرجة ا-عـ. وتدل الدرجة ا على الضعيف والدرجة r على الوسط والدرجة ب على الجيد

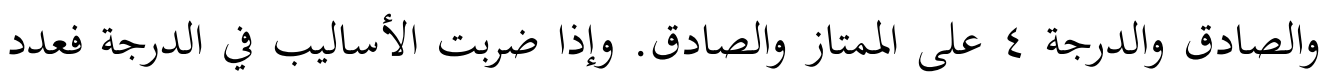

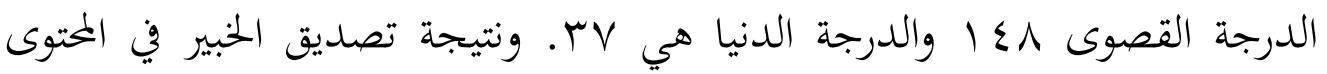

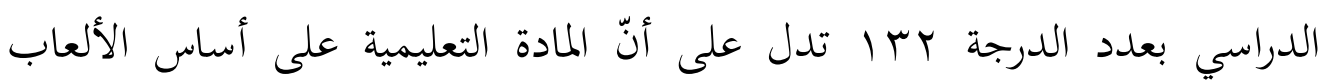
التقليدية على مستوى متتاز وصادق.

وأما بالنسبة إلى مقياس ليكرت (Likert) تتصورت النتيجة من تصديق الخبير في تصميم المادة التعليمية المطورة كما يلي: صورة ץ: مقياس ليكرت من نتيجة تصديق الخبير في تصميم المادة التعليمية المطورة

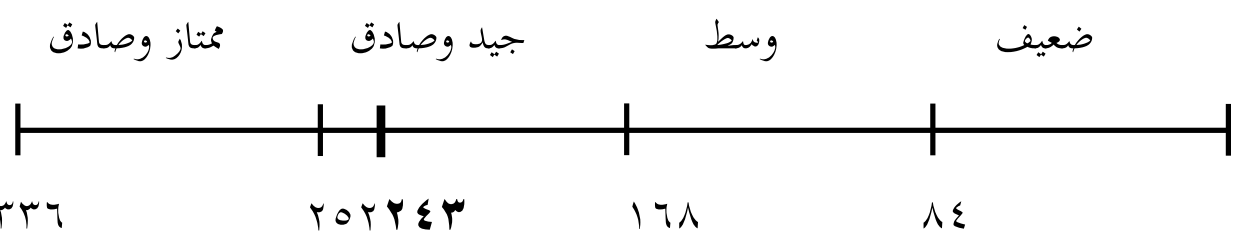


وفي أداة تصديق الخبير في تصميم المادة التعليمية £م أسلوبا بعرض الدرجة 1-ــ. وتدل الدرجة ا على الضعيف والدرجة ب على الوسط والدرجة ب على

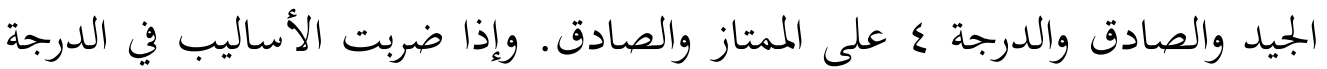
فعدد الدرجة القصوى بسا والدرجة الدنيا هي عـ. ونتيجة تصديق الخبير في

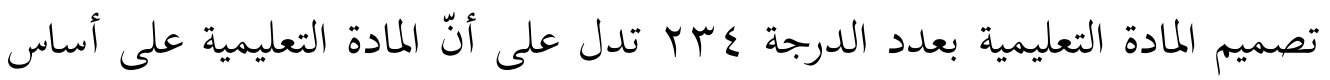
الألعاب التقليدية على مستوى جيد وصادق.

ومن هاتين النتيجتين حصلت المعلومات على أنّ المادة التعليمية على أساس ولى

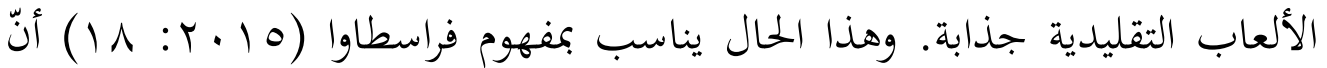

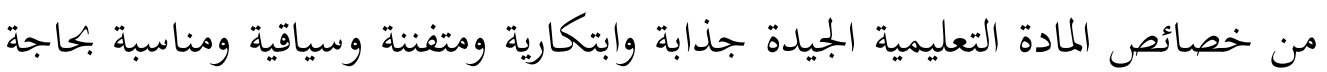

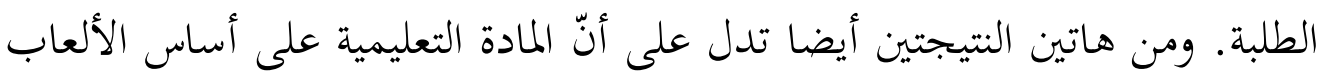
التقليدية صالح وصادق للاستخدام في تعليم اللغة العربية. التجربة وبعد الحصول على المادة التعليمية والقيام بتصديق الخبراء والتصحيح،

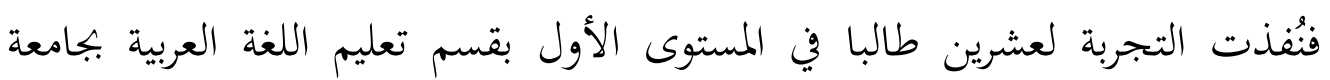
التنوير الإسلامية بوجونغارا ولمعلمة مادة اللغة العربية. وبعد القيام بالتجربة وُزعت الاستبانة لمم لمعرفة آرائهم وأجوبتهم نهو المادة التعليمية. وتتصورت نتعائج الاستبانة من الطلبة بالنسبة إلى مقياس ليكرت كما يلي: صورة ץ: مقياس ليكرت من نتيجة التجربة للطلبة

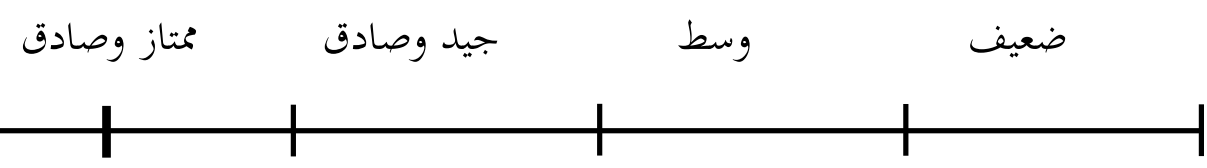
I. Y IIT. .
9.
7.
r..

وفي أداة التجربة للطلبة 1 أسلوبا بعرض الدرجة ا-هـ. وتدل الدرجة |

على الضعيف والدرجة r على الوسط والدرجة ب على الجيد وصادق والدرجة ع

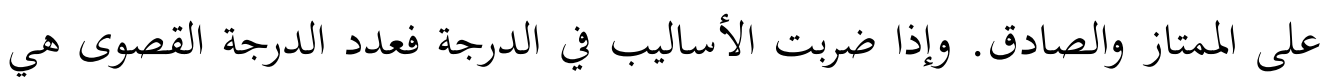

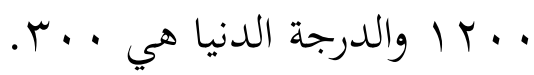




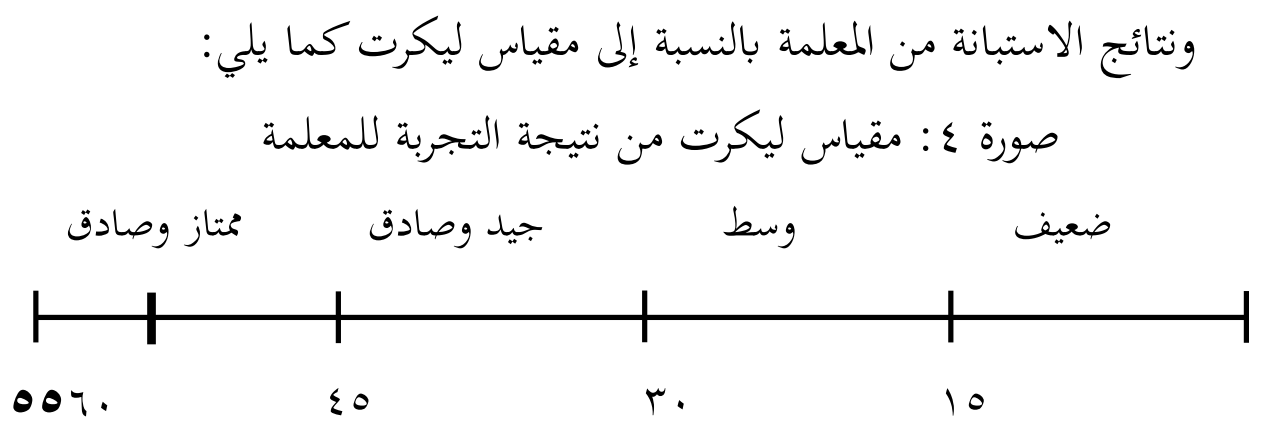

وفي أداة التجربة للمعلمة ه 1 أسلوبا بعرض الدرجة ا-عــ وتدل الدرجة ا

على الضعيف والدرجة r على الوسط والدرجة ب على الجيد والصادق والدرجة ع على الممتاز والصادق. وإذا ضربت الأساليب في الدرجة فعدد الدرجة القصوى هي .

ونتيجة التجربة للطلبة بعدد الدرجة اب • | ونتيجة التجربة للمعلمة بعدد الدرجة هـ تدلان على أنّ المادة التعليمية على أساس الألعاب التقليدية على مستوى محتاز وصادق. ومن هاتين النتيجتين حصلت المعلومات على أنّ المادة التعليمية على أساس الألعاب التقليدية لطلبة قسم تعليم اللغة العربية بجامعة التنوير الإسلامية بوجونغارا صالح وصادق للاستخدام في عملية التعليم.

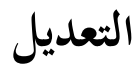

الخطوة الأخيرة في تطوير المادة التعليمية هي التعديل. وفي هذه الخطوة قامت

الباحثة بتصحيح المادة التعليمية وتحسينها حسب المداخلات من قبل الخبير ومعلمة المادة والطلبة. وأما المداخلات من الخبير في الحتوى الدراسي المطور والخبير في تصميم المادة التعليمية المطورة فهي: (1) تحسين الأخطاء الكتابية في صفحة الغلاف؛ (r) تفصيل كل المهارات في دليل استخدام الكتاب؛ (r) كتابة قائمة المحتويات باللغة العربية؛ (ع) مراجعة أخطاء استخدام المصطلاححات في المعجم؛ و (0) تكثير عدد التدريبات. وبعد القيام بتصحيح المادة التعليمية فُنفذ التحسين عليها فهي: (1) تحسين أخطاء الكتابة في صفحة الغلاف؛ (r) تفصيل كل المهارات في دليل استخدام الكتاب؛ (r) تغيير كتابة قائمة المحتويات باللغة العربية؛ (ع) تحسين أخطاء 
استخدام المصطلاحات؛ و(0) تكثير نوع التدريبات في كل المواد. وفي عملية التعديل ما وجدت المداخلات من نتيجة التجربة للطلبة ومعلمة مادة اللغة العربية. وفقا لذلك الحال فهذا الكتاب التعليمي صالح وصادق ومتوافق مع حاجة المستخدِم.

\section{مناقشة نتائج البحث}

إن أنشطة التعليم ليست جامدة، ولكنها عملية ديناميكية وابتكارية وتقدمية

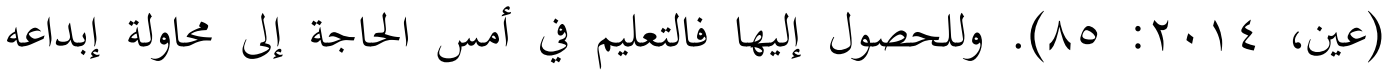

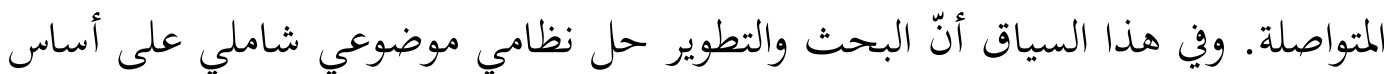
إنتاج التعليم الجودي مع تقدم العلوم والتكنولوجيا.


إنتاج معين وبتربة فعالية ذلك الإنتاج. وكيفية عمل هذا البحث لا تتعلق مع مشكلات

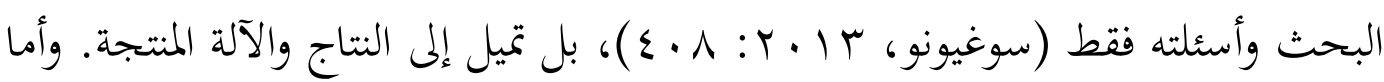

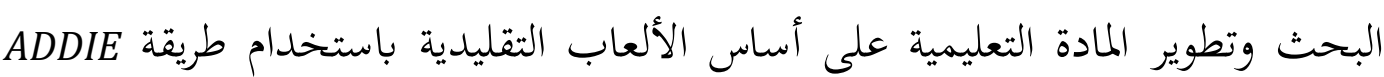
فيرتبط على الدراسة المبدئية، والتصميم، والتطوير، والتجربة، والتعديل).

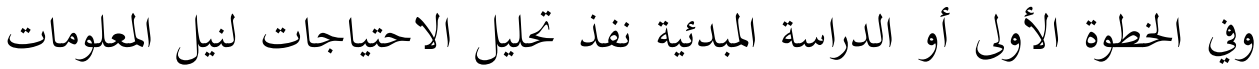
الأولى. وللحصول عليها فُنفذت المقابلة مع مدرسة اللغة العربية لمادة اللغة العربية الأولى لهلى بقسم تعليم اللغة العربية وهي الأستاذة نور ليلى رحمواتي الماجستير وطلبة قسم تعليم اللغة العربية. والمقابلة هي إحدى الخطوات في جمع البيانات. قال أريكونطا أنّ المقابلة هي

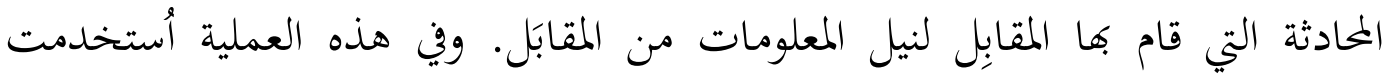
استمارة المقابلة لنيل المعلومات الأولى من جهة عملية التعليم والمادة المستخدمة والكفاءة المعلَّمة وإتاحة الفرصة للطلبة وكفاءة الطلبة.

وفقا لنتائج المقابلة المذكورة فقبل تصميم المادة التعليمية وجب على القيام بملاحظة المراجع التي تتوافق مع الحاجة ثم تعيين الموضوعات المناسبة مع حاجة الطلبة 
والمعلمة. وهذا الحال يتناسب مع رأي أشعري في دارماواتي أنّ حاجة الطلبة وأهدافهم معيار حيوي في عملية إعداد المادة التعليمية. إضافة إلى نتائج تحليل الاحتياجات من المعلمة والطلبة فيما يتعلق بالموضوع في المادة التعليمية فتحتوي على سبعة موضوعات، وهي: التعارف، وفي السوق، والبحث عن العمل، ووكالة السفر، ويف البنك، وفي بيت المال، والفن اليدوي.

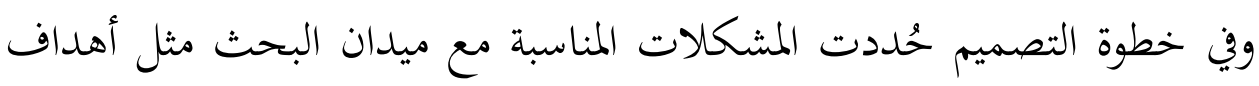
التعليم أو الكفاءة الأساسية، والمؤشرات، ومحتوى المادة التعليمية، ونموذج عرض المواد،

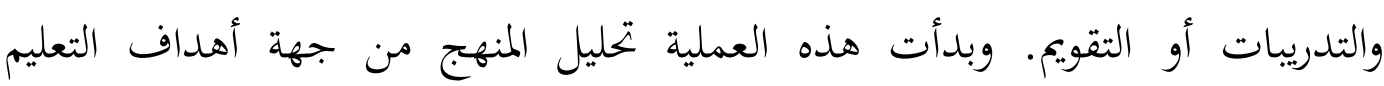
والكفاءة الأساسية والمؤشرات في مادة اللغة العربية بقسم تعليم اللغة العربية. وتحليل المنهج أمر ضروري لإجابة ثلاثة أحوال وهي: (1) هل الكفاءة التي سيحصل عليها

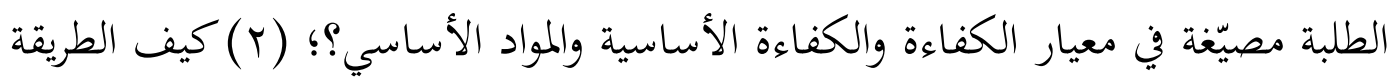
للحصول عليها التي فصّلت في أنشطة التعليم وعدد الوقت وأدوات أو مصدر التعليم المستخدم كيف معرفة حاصل الكفاءة بوجود إعداد المؤشرات كالمرجع في تعيين الجنس


وفقا لتحليل المنهج وأهداف التعليم المتبعة فالخلاصة أنّ أهداف تعليم اللغة

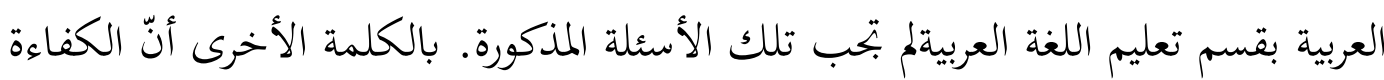

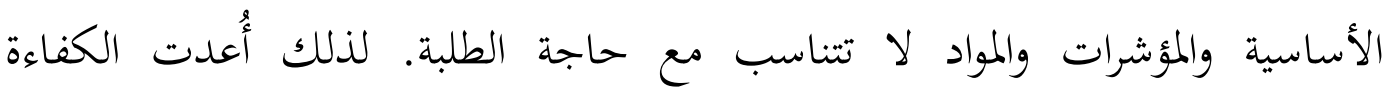
الأساسية والمؤشرات والمواد والتقويم بصفته الأسئلة الجلديدة المكتوبة في الكتاب التعليمي. وهذه الكفاءة المرادة تتناسب مع السياق في مجال تعليم اللغة العربية .

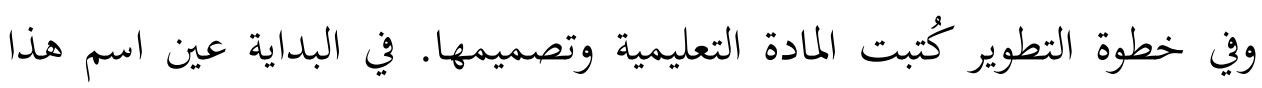
الكتاب وهو "اللغة العربية للاقتصاد الشرعي، الكتاب التعليمي لطلبة الجامعة بقسم تعليم اللغة العربية ". تم أستخدم تنفيذ Microsoft Word في تصميم محتوى الكتاب لتعبيه وتنفيذ Photoshop في تصميم غلافه. والثانية القيام بتعيين نظام الكتاب التعليمي الذي يحتوي على الغلاف والمقدمة ودليل استخدام الكتاب والكفاءة الأساسية والمؤشرات وقائمة المحتويات والمواد (أنشطة 
تعليم المفردات والكلام والقراءة والكتابة ثم التدريبات). و المواد تحتوي على سبعة موضوعات، وهي التعارف، وفي السوق، والبحث عن العمل، ووكالة السفر، وين البنك، وفي بيت المال، والفن اليدوي. وبعد تعيين نظام الكتاب التعليمي فالخطوة التالية هي القيام باختيار مقياس الكتاب المطور. واستخدم هذا الكتاب التعليمي مقياس B5. والمواد المكتوبة في أربعة أنشطة هي مواد جديدة مطورة واستخدمت الصور المأخوذة من الإنترنت والصور من الوثائق الذاتية. واستخدم وجه هذا الكتاب التعليمي تصميما ملوّنا. إنّ اختيار الموضوعات في هذا الكتاب مطابقة بنظرية التعليم السياقية. فقال مجيد أنّ التعليم السياقي هو عملية التعليم الكلي الذي يهدف لحفز الطلبة على فهم المواد التعليمسة والتربط عليها بسياقة حياتم اليومية إما السياق الشخصي والاجتماعي والثقافي حيث يملك الطلبة المعلومات والمهارات المطبقة من السياق الواحد إلى السياق الآخر. بالكلمة الأخرى أن الموضوعات في هذا الكتاب مناسبة بجهة الألعاب التقليدية، لأغّّا مناسبة بالأحوال والسياق المستخدم في بحال تعليم اللغة العربية. وكذلك في عملية التعليم أنّ كل الموضوعات مناسبة بخصائص التعليم السياقي.

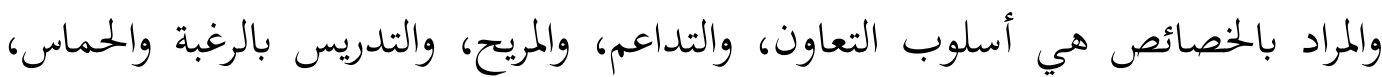

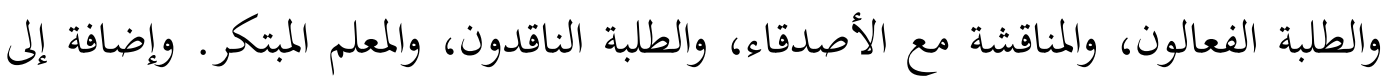
ذلك أنّ مواصفات هذا الكتاب مناسبة بالأهداف المرجوة في الأساس السياقي. وبعد انتهاء نموذج الكتاب التعليمي فالخطوة التالية هي تصديق الخبير. واستخدم هذا البحث والتطوير خبيرين، الخبير في المختوى الدراسي المطور والخبير في تصميم المادة التعليمية المطورة. ونُفّذ تصديق الخبير لمراجعة الانتاج الأوّل وإعطاء المداخلة لتحسينه. والمعنى أنّ القيام بتصديق الخبير لمعرفة درجة صادق المادة التعليمية الذي ارتكز على ولى ورلى

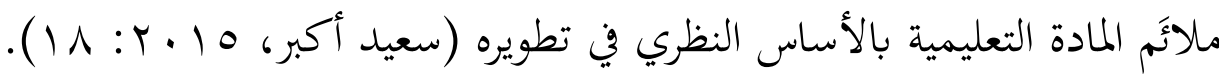
ومن نتيجتَي تصديق الخبير في المحتوى الدراسي المطور والحبير في تصميم المادة

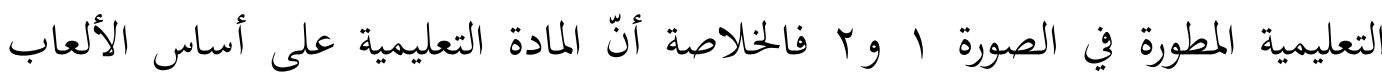


التقليدية جذابة. وهذا الحال يتناسب معقول فراسطاوا أنّ من خصائص المادة التعليمية الجيدة جذابة وابتكارية ومتفننة وسياقية ومناسبة بحاجة الطلبة.


المادة التعليمية على أساس الألعاب التقليدية لطلبة قسم تعليم اللغة العربية جامعة التنوير

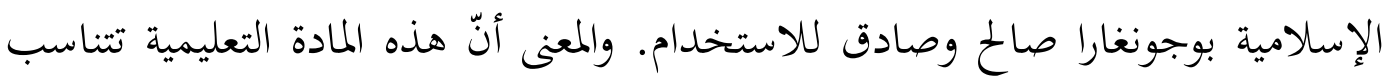
مع حاجة الطلبة والمعلمة في عملية تعليم اللغة العربية.

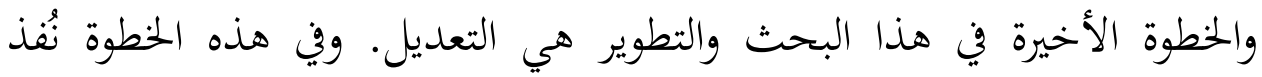
تصحيح المادة التعليمية وتحسينها حسب المداخلات من قبل الخبير. وبعد القيام بتصحيح المادة التعليمية فُنْفّذ التحسين عليها فهي: (1) تحسين أخطاء الكتابة في صفحة الغلاف؛ (r) تفصيل كل المهارات في دليل استخدام الكتاب؛ (r) تغيير كتابة قائمة المحتويات باللغة العربية؛ ؟() تحسين أخطاء استخدام المصطلاحات؛ و(0) تكثير نوع التدريبات في كل المواد. وفي عملية التعديل ما وجدت المداخلات من نتيجة التجربة للطلبة ومعلمة مادة اللغة العربية.

\section{الحخلاصة}

إن المواد التعليميـة لهـا أهميـة كبيرة لـدى المدرسـين في تعليم اللغـة العربية، لأهـا تساعدهم في تعليمهم، وكذلك لها أثر كبير لدى الطلبة في تعلم اللغة العربية وتسـاعد الطلبة في مجتمعاهم التي ينتمون إليها لتكوين الاستعدادات والقدرات للتعبير عن أفعالمم

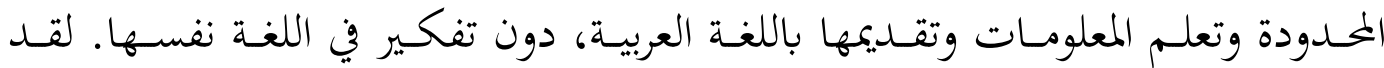
استخدم مصطلح الألعاب في تعليم اللغة، لكي يعطى مجالا واسعا من الأنشطة الفصلية التي تستخدم في التدريب على استخدام اللغة وممارستها، وهذه الألعاب تخضع لإشراف

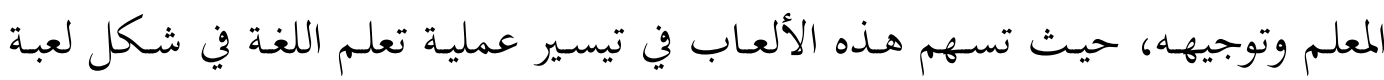
محكومة بقواعد معينة. فالمادة التعليمية المطورة على أساس الألعاب التقليدية تضم على المواد والأنشطة

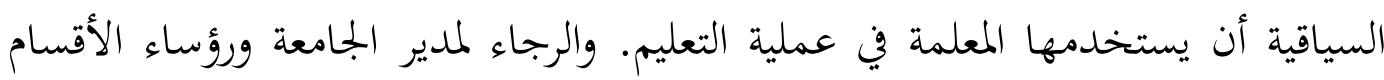


أن يشجع المعلمين للقيام بتطور المادة التعليمية الجيدة. ينبغي للمعلم أن يتعمق في أهداف تعليم اللغة العربية لأغراض خاصة وخصائصها ومحتوياتًا حسب الحاجات وتقترح الباحثة على أن يستفيد المعلم من المادة المطورة في عملية التعليم. إن هذه المادة التعليمية على أساس الألعاب التقليدية مازالت محددة بثلاثة عشرة موضوعا، ولم بجرب الباحثة فعاليتها في مجتمع البحث الأكبر. فينبغي للباحثين الآخرين أن يطوروا الموضوعات الآخرى حسب الحاجات ويجربوا هذه المادة التعليمية في ججتمع البحث الأكبر لمعرفة مدى فعاليتها. وفقا لذلك الحال فهذا الكتاب التعليمي صالح وصادق ومتوافق مع حاجة المستخدِم.

\section{المراجع}

أحمد، فوزي طه ورجب أحمد الكلزة. المناهج المعاصرة. الأسكندرية: منشأة المعارف، $. r . .0$

بحر الدين، أوريل. مهارات التدربس. مالانق: مطبعة جامعة مولانا مالك إبراهيم

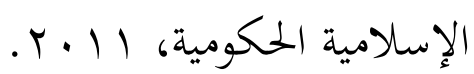

دارماواتي، رضى. تطوير مواد مهارة القراءة لأغراض أكاديمية خاصة لقسم التببية الإسلامية عبر تطبيقات Google Apps for Education دافعية التعلم والتحصيل الدرامي (بالتطبيق على جامعة أنتاساري الإسلامية الحلكومية بكاليمانتان الجنوبية. مجموعة بكوث اللغة العربية أساس الثقافة الإنسانية. مالانق: مطبعة جامعة مولانا مالك إبراهيم الإسلامية الحكومية، 10 شلبي، مصطفى. تعليم اللغة العربية والتوبية الدينية الإملامية. القـاهرة: دار الشـمس، . T... عيساني، عبد المجيد. نظريات التعلم وتطبيقاها في علوم اللغة. القاهرة: دار الكتاب

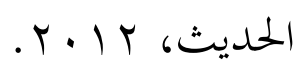

الغالي، ناصر عبد الله وعبد الحميد عبد الله. أسس إعداد الكتب التعليمية لغير الناطقين

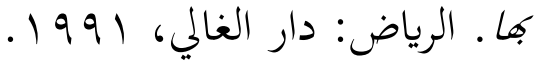




$$
\begin{aligned}
& \text { الهدى، مفتاح. تعليم اللغة العربية لأغراض خاصة (بكربة جامعة مولانا مالك إبراهيم } \\
& \text { الإسلامية الحلكومية مالانق إنلونيسيا). مجموعة بجوث اللغة العربية أساس الثقافة } \\
& \text { الإنسانية. مالانق: مطبعة جامعة مولانا مالك إبراهيم الإسلامية الحكومية، } \\
& . r .10
\end{aligned}
$$

Ainin, Moh. Metodologi Penelitian Peningkatan Kualitas Pembelajaran Bahasa Arab. Malang: CV. Bintang Sejahtera, 2014.

Ainin. Metodologi Peneltian Bahasa Arab. Surabaya: Hilal, 2010.

Akbar, Sa'dun. Instrumen Perangkat Pembelajaran. Bandung: PT. Remaja Rosdakarya, 2015.

Al Gali, Abdullah dan Abdullah, Abdul Hamid. MenyusunBahan Ajar Bahasa Arab. Padang: Akademia, 2012.

Asrori, Imam. Strategi Belajar Bahasa Arab. Malang: Misykat, 2011.

Branch, Robert Maribe. Instructional Design: The ADDIE Approach. New York: Springer, 2009.

Ghazali, Syukur. Pembelajaran Keterampilan Berbahasa dengan Pendekatan Komunikatif-Interaktif. Bandung: PT. Refika Aditama, 2010.

Komara, Endang. Belajar dan Pembelajaran Interaktif. Bandung: PT. Refika Aditama, 2014.

Lestari, Ika. Pengembangan Bahan Ajar Berbasis Kompetensi. Padang: Akademia, 2013.

Majid, Abdul. Strategi Pembelajaran. Bandung: PT. Remaja Rosdakarya, 2013.

Prastowo, Andi. Panduan Kreatif Membuat Bahan Ajar Inovatif. Yogyakarta: Diva Press, 2015.

Rusman. Model-model Pembelajaran. Jakarta: PT. Raja Grafindo Persada, 2013.

Sitepu, Bintang Petrus. Penulisan Buku Teks Pelajaran. Bandung: PT. Remaja Rosdakarya, 2012.

Sugiyono. Metode Penelitian Pendidikan Pendekatan Kuantitatif, Kualitatif dan $R \& D$. Bandung: Alfabeta, 2013.

Suja'i. Inovasi Pembelajaran Bahasa Arab: Strategi dan Metode Pengembangan Kompetensi. Semarang: Walisongo, 2008. 
Sukmadinata, Nana Syaodih. Metode Penelitian Pendidikan. Bandung: Remaja Rosdakarya, 2007.

Suyono dan Harianto. Belajar dan Pembelajaran. Bandung: PT. Remaja Rosdakarya, 2012.

Zainuddin, Radliyah. Metodologi dan Strategi Alternatif Pembelajaran Bahasa Arab. Yogyakarta: Pustaka Rihlah Grup, 2005. 